vance to the question of its creation, just as whether an artist's line has a beginning and an end, or instead forms a circle with no end, has no relevance to the question of its being drawn.

Of course, if the no-boundary proposal is correct, it does have interesting implications for the beauty, elegance and simplicity of the Universe God did create. Hawking draws the conclusion that God "had no freedom at all to choose initial conditions", but this is debatable. When I was defending Hawking's proposal to a small group of gravitational theorists in 1982, Bryce DeWitt expressed this view by saying, "You do not want to give God any freedom at all". However, Karel Kuchař quickly rejoined, "But that's His choice". In other words, even if we correctly hypothesize which state God chose for the Universe, that would in no way eliminate the freedom He may have had in making that choice. Choosing the noboundary state and then actually carrying out the immense task of the creation of the Universe in this state is a far cry from Carl Sagan's claim in his introduction to the book of "nothing for a Creator to do".

So although the final quarter of $A$ Brief History of Time is most interesting, it is also controversial. Nonetheless, the book should be acquired by anyone seeking to learn about some of the latest ideas and speculation in cosmology, and to gain familiarity with the insights that have sprung from the extraordinary mind of Stephen Hawking.

Don N. Page is Professor of Physics at The Pennsylvania State University, University Park, Pennsylvania 16802, USA.

\section{... and Weinberg revisited}

\section{David Layzer}

The First Three Minutes: A Modern View of the Origin of the Universe. By Steven Weinberg. Basic Books: 1988. Pp.198. Hbk \$16.95; pbk \$7.95. In Britain the book is out of print.

Big Bang, Big Bounce: How Particles and Fields Drive Cosmic Evolution. By Iosif L. Rozental. Translated by Juri Estrin. Springer-Verlag: $1988 . \quad P p .129 . \quad P b k$ DM58, £22, \$35.

AFTER eleven years, The First Three Minutes remains, in my opinion, the best introduction to cosmology for nonmathematical readers. Galileo, the first and most successful popularizer of cosmological theories, pinpointed the central difficulty of such an enterprise when he remarked (in another context) that the book of nature is written in the language of mathematics. Like poetry, mathematics always loses something in translation. Yet Weinberg manages not only to capture some of his subject's deepest ideas in clear, lively prose but also to communicate a personal vision of what science is and how it grows. This new edition is a reprint of the original text and of an afterword added in 1982 .

Two main themes are intertwined in the book: the history of the early Universe and the history of modern cosmology. Weinberg pays special attention to the historical antecedents of Arno Penzias and Robert Wilson's accidental discovery, in 1965, of the cosmic radiation background, and he devotes an entire chapter to the question of why astronomers didn't look for (and find) the background radiation earlier.

The text of The First Three Minutes and the seven-page afterword of 1982 are themselves interesting historical documents. In 1976, the 'standard model' of the early Universe, which interprets the radiation background as the fossil remnant of a primordial fireball, seemed firmly entrenched. It explained the blackbody character of the background radiation and it made predictions of the primordial abundances of helium and deuterium that seemed to be consistent with astronomical evidence. It did not, however, explain why the Universe is clumpy or why it contains roughly a thousand million photons for every nucleon. Clumpiness and the photon/nucleon ratio had to be put in 'by hand' - postulated as initial conditions. But most cosmologists were confident that these problems would eventually be solved. In 1976 Weinberg saw only one potential threat to the standard model, "one great uncertainty that hangs [over it] like a dark cloud": although the radiation background is now uniformly bright in all directions, there is no direct evidence that it was uniform and isotropic at very early times, as the standard model assumes.

By 1982, when Weinberg wrote his afterword, the picture had changed. New astronomical data and refined analyses of old data implied that if the standard model is correct, only a small fraction (now estimated at one per cent or less) of the mass in the Universe resides in ordinary matter. The bulk of the mass must reside in particles for whose existence there is as yet no independent experimental evidence. Meanwhile, Weinberg and other particle physicists, following up an early suggestion by Andrei Sakharov, had constructed a new theoretical foundation for the standard model. They conjectured that the Universe initially contained equal numbers of particles and antiparticles, and argued that the slight excess of particles needed to account for the observed photon/baryon ratio could have been pro- duced by baryon-nonconserving processes during a critical early period of the cosmic expansion. Baryon-nonconserving processes are permitted by unified theories of the strong and electroweak interactions. These theories also allow neutrinos to have small but finite mass. Could the missing mass be in the form of massive neutrinos? In 1982 the answer seemed to be yes.

Since 1982 the picture has changed again. Massive neutrinos have been ruled out as carriers of the missing mass, and no adequate replacement for them has emerged. The simplest unified theory of the strong and electroweak interactions has been found to conflict with experimental lower limits on the lifetime of the proton. Alan Guth's inflationary hypothesis, put forward in 1981 and described briefly in Weinberg's afterword, has solved some the problems - notably the magnetic-monopole problem - but it has created others. Increasingly stringent observational limits on small-scale angular variations in the brightness of the radiation background have made it increasingly difficult to reconcile the hypothesis of a primordial fireball with the observed clumpiness of the astronomical universe. And the standard model has split, at its base, into a dozen or so competing hypotheses, each more speculative than the last.

One of these hypotheses - that the Universe is made up of non-interacting 'metagalaxies', each with its own physical constants and laws - forms the central theme of Big Bang, Big Bounce, a book intended to introduce readers who "have taken courses in physics and mathematics at the undergraduate or graduate level" to some elementary concepts of particle physics and cosmology. In his preface, Rozental praises Weinberg's book, and his text is intended as a kind of sequel to The First Three Minutes.

I found the sections on particle physics clear and interesting. The sections that deal with observational and theoretical cosmology are less satisfactory. For the most part they are superficial and perfunctory, and some of them - for example, the discussion of 'Newtonian cosmology', a topic that Weinberg treats very well are seriously misleading. As for the translation, it preserves the flavour of the original Russian so successfully that one is hardly aware that one is reading English.

David Layzer is the Donald H. Menzel Professor of Astrophysics at Harvard University, 60 Garden Street, Cambridge, Massachusetts 02138, USA.

- Another classic example of popular exposition in cosmology, Arthur Eddington's The Expanding Universe, which was first published in 1933, has recently been re-issued in paperback by Cambridge University Press. Price is $£ 7.95, \$ 12.95$. 